\title{
RNase $E$ action at a distance: degradation of target mRNAs mediated by an Hfq-binding small RNA in bacteria
}

\author{
Teppei Morita and Hiroji Aiba ${ }^{1}$ \\ Faculty of Pharmaceutical Sciences, Suzuka University of Medical Sciences, Suzuka, Mie 513-0816, Japan
}

A major class of bacterial small RNAs (sRNAs), along with RNA-binding protein Hfq and endoribonuclease RNase E, acts on target mRNAs through base-pairing, leading to translational repression and rapid degradation of the mRNAs. In this issue of Genes \& Development, Prévost and colleagues (pp. 385-396) demonstrate by using the well-characterized SRNA RyhB that RNase E cleavage at sites distal from the pairing region triggers degradation of target mRNAs. The study has provided an important insight into the initial events of sRNAinduced degradation of target mRNAs.

Regulation of gene expression by RNA molecules was known in prokaryotes far before the discovery of microRNAs (miRNAs) and siRNAs in eukaryotes. Since the serendipitous discovery of MicF, which is involved in the down-regulation of the expression of $o m p F$, a gene that encodes a major outer membrane porin of Escherichia coli (Mizuno et al. 1984), a number of regulatory RNAs have been shown to be widely involved in the regulation of gene expression in bacteria. Among them, the Hfqbinding small RNAs (sRNAs), which are functionally analogous to eukaryotic miRNAs, have been most extensively studied (for reviews, see Waters and Storz 2009; Gottesman and Storz 2010). These sRNAs, which are generally transcribed under specific physiological conditions, act by pairing to target mRNAs and regulating their translation and stability, in many cases negatively, with the help of the RNA-binding protein Hfq. Hfq facilitates the pairing and stabilization of sRNAs, with the major outcome being translational repression and rapid degradation of the target mRNAs.

The mechanism by which sRNAs lead to translational repression is rather simple and fairly well understood. sRNAs mostly bind to the translation initiation region of target mRNAs and occlude the ribosome-binding site (RBS) (for reviews, see Waters and Storz 2009; Gottesman

[Keywords: RyhB; small RNA; RNase E; RNA degradosome; mRNA decay; translation initiation; Hfq]

${ }^{1}$ Corresponding author.

E-MAIL aiba@suzuka-u.ac.jp; FAX 81-59-368-1271.

Article is online at http://www.genesdev.org/cgi/doi/10.1101/gad.2030311. and Storz 2010). However, the mechanism of sRNAmediated degradation of target mRNAs is apparently complicated and is only partially understood. Based on the studies on a few sRNAs, several notable features have been uncovered (for review, see Aiba 2007). First, a major endoribonuclease, RNase E, is responsible for the sRNAmediated destabilization of target mRNAs (Massé et al. 2003; Vanderpool and Gottesman 2004; Kawamoto et al. 2005). Second, sRNAs paired with target mRNAs are degraded together in an RNase E-dependent manner (Massé et al. 2003). Third, Hfq, along with Hfq-binding sRNAs, binds to RNase E, resulting in the formation of specific ribonucleoprotein complexes that allow for the tethering of RNase E near the base-pairing region of target mRNAs (Morita et al. 2005). One of the unexplored questions is where and how the RNase E-dependent degradation of the mRNA-sRNA hybrid is initiated.

In this issue of Genes \& Development, Prévost et al. (2011) use the well-characterized sRNA RyhB to show that RNase E cleavage at sites distal from the pairing region triggers RyhB-mediated rapid degradation of target mRNAs. When RyhB pairs with the target mRNAs, ribosome loading onto the translation initiation region is blocked, and ribosomes already engaged in translation are eventually cleared from the downstream cleavage sites. This allows RNase E tethered at the pairing region to attack the cleavage sites, leading to the subsequent rapid degradation of target mRNAs. The work has thus provided an important insight into the initial events of RyhB-induced degradation of target mRNAs. However, it remains to be seen whether RNase E action at a distance is a general mechanism for the mRNA degradation mediated by Hfqbinding sRNAs.

In this perspective, we first summarize the general properties of Hfq-binding sRNAs, the roles of Hfq in sRNA function, and the two major regulatory outcomes of basepairing (translational repression and RNase E-dependent degradation of target mRNAs). We also discuss how these regulatory outcomes are mediated by a ribonucleoprotein complex consisting of RNase E, Hfq, and sRNAs. Then, we discuss the work in the Prévost et al. (2011) study and its implications for the role of Hfq-binding sRNAs in mRNA degradation, focusing on how the translational repression 
and RNase E-dependent degradation of target mRNAs mediated by sRNAs are interrelated.

\section{Hfq-binding sRNAs}

Hfq-binding sRNAs act through imperfect base-pairing with a single or multiple target mRNAs, regulating their translation and stability (for reviews, see Waters and Storz 2009; Gottesman and Storz 2010). In contrast to miRNAs that bind to the $3^{\prime}$ untranslated region (UTR) of target mRNAs, bacterial sRNAs bind to the 5' UTR of target mRNAs (Gottesman 2005). The translation initiation region in the target mRNAs is most often used for base-pairing, and the pairing therefore occludes the RBS and results in translational repression-although, in a few cases, the pairing occurs upstream of the RBS (for review, see Waters and Storz 2009). The sRNAs can also activate expression of the target mRNAs in some cases, by disrupting an inhibitory secondary structure that sequesters the RBS (for reviews, see Waters and Storz 2009; Gottesman and Storz 2010). Although the potential base-pairing between sRNAs and target mRNAs ranges from 10 to 30 nucleotides (nt), more limited core-pairing appears to be sufficient for regulation (Waters and Storz 2009). For example, a 14-nt sequence within the 31-nt potential pairing region of SgrS is sufficient to inhibit the target ptsG translation (Maki et al. 2010), and only four single mutations in SgrS significantly affect the down-regulation of ptsG mRNA (Kawamoto et al. 2006).

The RNA-binding protein Hfq, which was originally identified as a host factor required for the in vitro replication of the RNA phage $\mathrm{Q} \beta$ in E. coli, is extensively involved in the regulation of RNA metabolism (for review, see Valentin-Hansen et al. 2004). The involvement of Hfq in sRNA functions was first recognized during the study on translational repression of rpoS mRNA (encoding the alternative $\sigma$ factor RpoS) by OxyS RNA that is induced in response to oxidative stress in E. coli (Zhang et al. 1998). Subsequently, Hfq was shown to bind to and be necessary for the regulatory activity of a number of sRNAs (for reviews, see Gottesman 2004; Valentin-Hansen et al. 2004). Hfq resembles the Sm and Sm-like proteins involved in splicing and mRNA degradation in eukaryotic cells in that it forms a donut-shaped structure consisting of six identical 11-kDa subunits (Moller et al. 2002; Zhang et al. 2002). Hfq has been demonstrated to stimulate sRNA-mRNA base-pairing in vitro in several cases, such as OxyS-fhlA (Zhang et al. 2002), Spot42-galK (Moller et al. 2002), MicA-ompA (Rasmussen et al. 2005), and SgrS-ptsG (Kawamoto et al. 2006). A recent study has demonstrated that, in vitro, Hfq is no longer required for translational inhibition once base-pairing is achieved (Maki et al. 2008). However, all Hfq-binding sRNAs examined to date require $\mathrm{Hfq}$ for their regulatory functions in vivo. It is highly likely that the Hfq-mediated rapid association of sRNAs with target mRNAs is necessary to efficiently block ribosome loading to the RBS. Hfq promotes base-pairing by either changing the RNA structures; acting as an RNA chaperone, thereby allowing accessibility of two complementary RNAs; or binding to a given sRNA and its target
mRNAs simultaneously, thereby increasing the local concentrations of two RNAs (Storz et al. 2004). Besides facilitating base-pairing, Hfq also contributes to sRNA function by stabilizing sRNAs (for review, see Gottesman 2004).

\section{RNase E-dependent coupled degradation of target mRNAs and sRNAs}

Another major outcome of sRNA-mRNA pairing is the rapid degradation of target mRNAs. It is generally accepted that mRNA degradation in bacteria is initiated by endoribonucleolytic cleavage, resulting in mRNA fragments that are then degraded by $3^{\prime}-5^{\prime}$ exonucleases (for reviews, see Belasco 2010; Carpousis 2007). RNase E is a major endoribonuclease that is responsible for degradation of many RNAs in E. coli (for review, see Carpousis 2007). It forms the RNA degradosome, associating with polynucleotide phosphorylase (PNPase), RNA helicase $\mathrm{B}(\mathrm{RhlB})$, and the glycolytic enzyme enolase as the principle partners (for review, see Carpousis 2007). It is likely that both RNase $\mathrm{E}$ and PNPase within the degradosome act in a concerted fashion during the degradation of RNAs, while RhlB unwinds the secondary structure of target RNAs to permit access of RNase E and/or PNPase. The RNase E polypeptide is composed of an $\mathrm{N}$-terminal catalytic region and a C-terminal scaffold region responsible for the interaction with partner proteins (Carpousis 2007; Worrall et al. 2008).

sRNA-induced mRNA degradation was first observed in the study of RyhB and its target mRNAs. RyhB downregulates a number of genes that encode for iron-binding proteins, such as $\operatorname{sodB}$, which encodes for the superoxide dismutase enzyme in response to iron limitation (Massé and Gottesman 2002). RyhB causes a rapid degradation of target mRNAs in an RNase E-dependent manner (Massé et al. 2003). The RNase E-dependent rapid degradation of target mRNAs is probably a general feature of sRNA function (Morita et al. 2004, 2005). In addition, sRNAs were shown to be destabilized, depending on pairing with target mRNAs, also in an RNase E-dependent manner, suggesting that RNase E somehow specifically attacks the sRNA-mRNA duplex. (Massé et al. 2003). Furthermore, it was shown that the C-terminal scaffold region of RNase $\mathrm{E}$ is required for the coupled degradation of sRNAs and their target mRNAs (Massé et al. 2003; Morita et al. 2004).

\section{Ribonucleoprotein complexes consisting of sRNAs, Hfq, and RNase E}

A key discovery for understanding the mechanism of sRNA-mediated degradation of target mRNAs was that Hfq associates with RNase E through its scaffold region, and at least two sRNAs-SgrS and RyhB-associate with RNase E through Hfq (Morita et al. 2005). SgrS is another Hfq-binding sRNA; it is induced in response to the accumulation of glucose-phosphate, resulting in the RNase E-dependent destabilization of ptsG mRNA, which encodes for the glucose transporter IICB ${ }^{\text {Glc }}$ (Kimata et al. 2001; 
Morita et al. 2003; Vanderpool and Gottesman 2004). RyhB- and SgrS-mediated destabilization of target mRNAs is dependent on Hfq and the C-terminal scaffold region of RNase E (Kawamoto et al. 2005; Morita et al. 2004). The physical association of sRNAs/Hfq with RNase E explains how the functional cooperation of sRNAs, Hfq, and RNase $\mathrm{E}$ is achieved. A recent study has established that the subregion of RNase E overlapping the RhlB-binding site is responsible for the interaction with $\mathrm{Hfq}$, leading to the rapid degradation of target mRNAs mediated by sRNAs (Ikeda et al. 2011).

\section{RNase $E$ action at a distance}

Prévost et al. (2011) addressed where and how the initial cleavage by RNase E occurs in sRNA-mRNA duplexes. By analyzing a series of sodB-lac $Z$ transcriptional fusions in which various lengths of the $5^{\prime}$ portion of $\operatorname{sod} B$ transcript were fused to the $1 a c Z$, they defined a site, between +400 and +430 , far downstream from the pairing region, that is required for the RyhB-mediated degradation of the target sodB mRNA. This mRNA degradation was shown to be dependent on both RNase E and Hfq, suggesting that RNase E tethered near the $\operatorname{sodB}$ RBS cleaves the region between +400 and +430 . An AU-rich sequence within the $400-430$ region of $\operatorname{sodB}$ is cleaved by RNase $\mathrm{E}$ in vitro, and in vitro cleavage no longer occurred when the AU-rich sequence was mutated. More importantly, the mutation eliminated the RNase E-dependent rapid degradation of a sodB-lacZ fusion mRNA in vivo. Furthermore, a short sodB-lac $Z$ construct was RNase E-resistant but became sensitive to RNase E cleavage when the 30-nt section was added. This led Prévost et al. (2011) to conclude that the AU-rich sequence within the 400-430 region of $\operatorname{sodB}$ is sufficient to cause the RNase E-dependent rapid degradation mediated by $\mathrm{RyhB} / \mathrm{Hfq}$. They propose that RyhB induces an initial mRNA cleavage by RNase $\mathrm{E}$ at this distal site far downstream from the pairing region to destroy the target mRNA. Strictly speaking, it remains to be seen whether the cleavage at the distal AU-rich sequence by RNase E tethered at the base-pairing region is really an initial event for the rapid degradation of $\operatorname{sod} B$ mRNA mediated by RyhB, although it is surely the ratelimiting step.

mRNA cleavage at a distal site was observed in other RyhB target mRNAs, although the distance between the pairing site and the cleavage site varied depending on the target mRNA. This suggests that the mRNA cleavage at a distal site by RNase $\mathrm{E}$ is a general mechanism associated with sRNA-mediated rapid degradation of target mRNAs. Action at a distance is a widely used mechanism in the regulation of DNA and RNA functions. For example, it is well known that transcription of genes is regulated very often by transcription factors that bind to sites on the DNA at a considerable distance in both bacteria and eukaryotes (for review, see Ptashne 1986). Translation of mRNAs is stimulated by proteins bound at its $3^{\prime}$ polyA tail and inhibited by protein components within RISC (RNA-induced silencing complex) tethered at the 3' UTR through miRNAs in eukaryotes (for review, see Carthew and Sontheimer 2009). The major impact of the study by Prévost et al. (2011) is that it demonstrated that endoribonucleases can also act at a distance.

\section{Interplay between mRNA cleavage and translational repression}

How are the translational repression and rapid degradation of target mRNAs mediated by sRNAs interrelated? Theoretically, these two events could affect each other. Namely, translational inhibition can lead to destabilization of an mRNA by exposing it to ribonucleases, while mRNA degradation should certainly cause loss of translation by reducing mRNA concentration. A previous study on SgrS and RyhB demonstrated several key features of the interrelationship between translational repression and sRNA-mediated mRNA degradation (Morita et al. 2006). First, translational repression occurs without the RNase E-dependent degradation of mRNAs, and therefore is primarily responsible for the down-regulation of target mRNAs by sRNAs. Thus, the major role of the RNase E-dependent rapid degradation of mRNAs appears to be to make gene silencing irreversible and eliminate translationally inactive mRNAs, although mRNA degradation seem to contribute to the down-regulation of target mRNAs without blocking translation in one case (Pfeiffer et al. 2009). In addition, translational repression itself-by either $\mathrm{Hfq} / \mathrm{sRNAs}$ or a drug - is not sufficient for degradation, and recruitment of RNase $\mathrm{E}$ to the base-pairing region by Hfq/sRNAs is required for the RNase E-dependent rapid degradation of target mRNAs. The study by Prévost et al. (2011) confirmed and extended these previous conclusions. They showed, by introducing a stop codon far upstream of the cleavage site, that blocking translation alone is not sufficient to induce rapid degradation. In other words, RyhBmediated rapid mRNA degradation is an active outcome by a concerted action of RNase E, Hfq, and RyhB, rather than a passive secondary effect of blocking translation.

The work by Prévost et al. (2011) has provided an additional insight into the relationship between the translational repression and mRNA degradation mediated by sRNAs. This derives from the analysis of a series of SodBLacZ translational fusions. In contrast to the transcriptional fusions discussed above, all translational fusions were sensitive to RyhB-mediated degradation, even when the fusions did not harbor the identified RNase E site between +400 and +430 . This could be explained by assuming the presence of RNase E-sensitive sites within the LacZ ORF. If so, why are the additional cleavage sites resistant to RNase E in the sodB-lacZ transcriptional fusions? Although Prévost et al. (2011) did not extensively address this point in their study, the results provide further insight into the mechanism of RyhB-mediated mRNA cleavage at a distance. It is highly likely that the RyhB-mediated cleavage of target mRNAs at a distance occurs only when the translating ribosomes, which are expected to protect mRNAs from RNase attack, are cleared from the RNase E site. It is important to note that ribosomes are eventually cleared from the lac $Z$ portion in SodB-LacZ translational fusions when RyhB inhibits the 


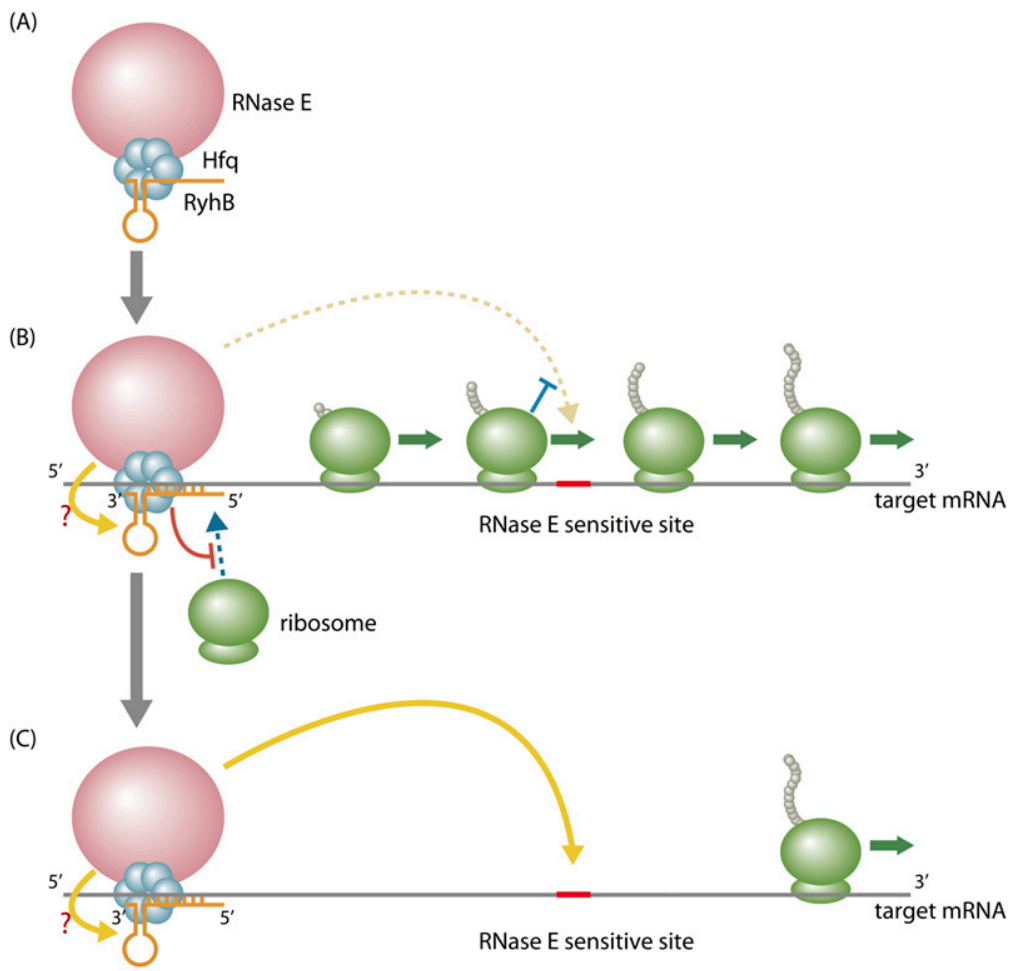

Figure 1. Model for RNase E action at a distance. (A) Hfq binds to the C-terminal scaffold region of RNase E. When RyhB is induced, it associates with Hfq and therefore RNase E, resulting in a specific ribonucleoprotein complex. $(B)$ The ribonucleoprotein complex acts on the target mRNAs: Hfq facilitates the pairing and RNase E is tethered at the pairing region. The pairing inhibits new ribosome loading, while translating ribosomes protect the downstream RNase E site (shown in red line) from the attack by RNase E. (C) RNase E tethered at the pairing region, but not freestanding RNase E/degradosome, efficiently attacks the RNase E site after the translating ribosomes have passed through. Whether RNase E tethered at the base-pairing region directly attacks the paired RyhB remains to be studied. new ribosome loading to the $\operatorname{sod} B$ RBS. On the other hand, the inhibition of $\operatorname{sod} B$ translation by RyhB does not affect the translation of the $l a c Z$ portion in transcriptional fusions because the $l a c Z$ translation occurs independently of $\operatorname{sod} B$ translation. Thus, the RNase E sites in the lacZ portion of the $\operatorname{sod} B-1 a c Z$ transcriptional fusions are protected by translating ribosomes. This is probably why the cleavage site within $1 a c Z$ is resistant to RNase E. In other words, the RyhB-mediated translational inhibition is necessary for RyhB-mediated mRNA cleavage. We emphasize that RNase E recruited to the target mRNA by Hfq/sRNAs-but not free RNase E-can efficiently attack the cleavage sites. Altogether, the data presented in the study by Prévost et al. (2011) lead to the model shown in Figure 1. When RyhB is induced, it associates with Hfq and RNase E. The resulting ribonucleoprotein complex acts on the target mRNAs to form the RyhB-mRNA hybrid along with Hfq and RNase $\mathrm{E}$, preventing the ribosome binding to the RBS of target mRNAs. Subsequently, ribosomes, which have already started translation of target mRNAs before the formation of the RyhB-mRNA hybrid, would pass through the RNase E-sensitive sites, allowing the action at a distance by RNase $\mathrm{E}$ tethered at the base-pairing region by $\mathrm{Hfq} / \mathrm{RyhB}$. Thus, blocking translation initiation is apparently a prerequisite to the efficient cleavage at the downstream RNase E-sensitive sites.

\section{Further questions}

While the work by Prévost et al. (2011) has uncovered key events in the initial stage of the sRNA-induced rapid degradation of target mRNAs, a number of questions remain to be answered. Among them, we discuss here several points for future studies. First, an intriguing question is why the identified RNase E-sensitive sites are attacked efficiently by RNase E tethered at the basepairing region, but not by the freestanding RNase E/ degradosome when translation is blocked. One possibility is that RNase E recruitment near the base-pairing region of an mRNA could simply increase the probability of RNase E attack. Alternatively, something more-other proteins and/or RNA structures_could be also required for the efficient cleavage. The in vitro reconstitution of RNase E-dependent rapid degradation of target mRNAs mediated by Hfq/sRNAs will be needed to answer this question. Second, the RNase E cleavage of mRNAs at a distance was shown only in the fusion genes. It is certainly important to show that this mechanism is indeed used in the degradation of natural target mRNAs of RyhB. Such analysis may also help define the critical features of RNase E sites. Third, the RNase E cleavage of mRNAs at a distance, mediated by sRNAs, was shown, thus far, only for the targets of RyhB. While this is likely also to be true for other Hfq-binding sRNAs and their targets, this remains to be shown. Finally, we also need to know whether the sRNAs paired with target mRNAs themselves are attacked directly by RNase E tethered at the base-pairing region.

\section{Acknowledgments}

We thank Susan Gottesman for comments on the manuscript.

\section{References}

Aiba H. 2007. Mechanism of RNA silencing by Hfa-binding small RNAs. Curr Opin Microbiol 10: 134-139. 
Belasco JG. 2010. All things must pass: Contrasts and commonalities in eukaryotic and bacterial mRNA decay. Nat Rev Mol Cell Biol 11: 467-478.

Carpousis AJ. 2007. The RNA degradosome of Escherichia coli: An mRNA-degrading machine assembled on RNase E. Annu Rev Microbiol 61: 71-87.

Carthew RW, Sontheimer EJ. 2009. Origins and mechanisms of miRNAs and siRNAs. Cell 136: 642-655.

Gottesman S. 2004. The small RNA regulators of Escherichia coli: Roles and mechanisms. Annu Rev Microbiol 58: 303-328.

Gottesman S. 2005. Micros for microbes: Non-coding regulatory RNAs in bacteria. Trends Genet 21: 399-404.

Gottesman S, Storz G. 2010. Bacterial small RNA regulators: Versatile roles and rapidly evolving variations. Cold Spring Harb Perspect Biol 1: 1-16.

Ikeda Y, Yagi M, Morita T, Aiba H. 2011. Hfq binding at RhlBrecognition region of RNase $\mathrm{E}$ is crucial for the rapid degradation of target mRNAs mediated by sRNAs in Escherichia coli. Mol Microbiol 79: 419-432.

Kawamoto H, Morita T, Shimizu A, Inada T, Aiba H. 2005. Implication of membrane localization of target mRNA in the action of a small RNA: Mechanism of post-transcriptional regulation of glucose transporter in Escherichia coli. Genes Dev 19: 328-338.

Kawamoto H, Koide Y, Morita T, Aiba H. 2006. Base-pairing requirement for RNA silencing by a bacterial small RNA and acceleration of duplex formation by Hfq. Mol Microbiol 61: 1013-1022.

Kimata K, Tanaka Y, Inada T, Aiba H. 2001. Expression of the glucose transporter gene, ptsG, is regulated at the mRNA degradation step in response to glycolytic flux in Escherichia coli. EMBO J 20: 3587-3595.

Maki K, Uno K, Morita T, Aiba H. 2008. RNA, but not protein partners, is directly responsible for translational silencing by a bacterial Hfq-binding small RNA. Proc Natl Acad Sci 105: 10332-10337.

Maki K, Morita T, Otaka H, Aiba H. 2010. A minimal basepairing region of a bacterial small RNA SgrS required for translational repression of ptsG mRNA. Mol Microbiol 76: 782-792.

Massé E, Gottesman S. 2002. A small RNA regulates the expression of genes involved in iron metabolism in Escherichia coli. Proc Natl Acad Sci 99: 4620-4625.

Massé E, Escorcia FE, Gottesman S. 2003. Coupled degradation of a small regulatory RNA and its mRNA targets in Escherichia coli. Genes Dev 17: 2374-2383.

Mizuno T, Chou MY, Inouye M. 1984. A unique mechanism regulating gene expression: Translational inhibition by a complementary RNA transcript (micRNA). Proc Natl Acad Sci 81: 1966-1970.

Moller T, Franch T, Hojrup P, Keene DR, Bachinger HP, Brennan RG, Valentin-Hansen P. 2002. Hfq: A bacterial Sm-like protein that mediates RNA-RNA interaction. Mol Cell 9: 23-30.

Morita T, El-Kazzaz W, Tanaka Y, Inada T, Aiba H. 2003. Accumulation of glucose 6-phosphate or fructose 6-phosphate is responsible for destabilization of glucose transporter mRNA in Escherichia coli. J Biol Chem 278: 15608-15614.

Morita T, Kawamoto H, Mizota T, Inada T, Aiba H. 2004. Enolase in the RNA degradosome plays a crucial role in the rapid decay of glucose transporter mRNA in the response to phosphosugar stress in Escherichia coli. Mol Microbiol 54: 1063-1075.

Morita T, Maki K, Aiba H. 2005. RNase E-based ribonucleoprotein complexes: Mechanical basis of mRNA destabilization mediated by bacterial noncoding RNAs. Genes Dev 19: $2176-2186$.
Morita T, Mochizuki Y, Aiba H. 2006. Translational repression is sufficient for gene silencing by bacterial small noncoding RNAs in the absence of mRNA destruction. Proc Natl Acad Sci 103: 4858-4863.

Pfeiffer V, Papenfort K, Lucchini S, Hinton JC, Vogel J. 2009. Coding sequence targeting by MicC RNA reveals bacterial mRNA silencing downstream of translational initiation. Nat Struct Mol Biol 16: 840-846.

Prévost K, Desnoyers G, Jacques J-F, Lavoie F, Massé E. 2011. Small RNA-induced mRNA degradation achieved through both translation block and activated cleavage. Genes Dev (this issue). doi: 10.1101/gad.2001711.

Ptashne M. 1986. Gene regulation by proteins acting nearby and at a distance. Nature 322: 697-701.

Rasmussen AA, Eriksen M, Gilany K, Udesen C, Franch T, Petersen C, Valentin-Hansen P. 2005. Regulation of ompA mRNA stability: The role of a small regulatory RNA in growth phase-dependent control. Mol Microbiol 58: 14211429.

Storz G, Opdyke JA, Zhang A. 2004. Controlling mRNA stability and translation with small, noncoding RNAs. Curr Opin Microbiol 7: 140-144.

Valentin-Hansen P, Eriksen M, Udesen C. 2004. The bacterial Sm-like protein Hfq: A key player in RNA transactions. Mol Microbiol 51: 1525-1533.

Vanderpool CK, Gottesman S. 2004. Involvement of a novel transcriptional activator and small RNA in post-transcriptional regulation of the glucose phosphoenolpyruvate phosphotransferase system. Mol Microbiol 54: 1076-1089.

Waters LS, Storz G. 2009. Regulatory RNAs in bacteria. Cell 136: 615-628.

Worrall JA, Gorna M, Crump NT, Phillips LG, Tuck AC, Price AJ, Bavro VN, Luisi BF. 2008. Reconstitution and analysis of the multienzyme Escherichia coli RNA degradosome. I Mol Biol 382: 870-883.

Zhang A, Altuvia S, Tiwari A, Argaman L, Hengge-Aronis R, Storz G. 1998. The OxyS regulatory RNA represses rpoS translation and binds the Hfq (HF-I) protein. EMBO $J$ 17: 6061-6068.

Zhang A, Wassarman KM, Ortega J, Steven AC, Storz G. 2002. The Sm-like Hfq protein increases OxyS RNA interaction with target mRNAs. Mol Cell 9: 11-22. 


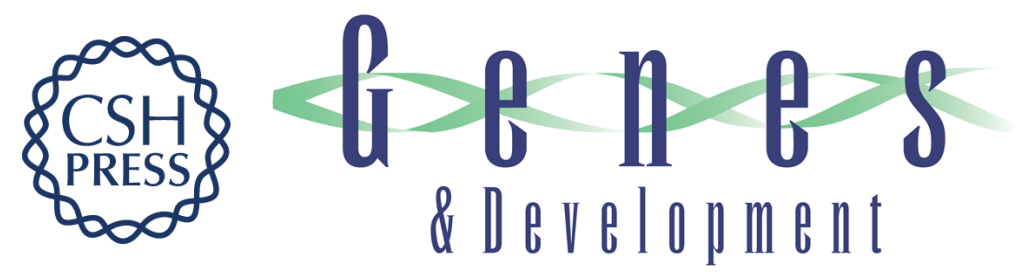

\section{RNase E action at a distance: degradation of target mRNAs mediated by an Hfq-binding small RNA in bacteria}

Teppei Morita and Hiroji Aiba

Genes Dev. 2011, 25:

Access the most recent version at doi:10.1101/gad.2030311
Related Content Small RNA-induced mRNA degradation achieved through both translation block and activated cleavage
Karine Prévost, Guillaume Desnoyers, Jean-François Jacques, et al.
Genes Dev. February , 2011 25: 385-396
References This article cites 31 articles, 10 of which can be accessed free at:
http://genesdev.cshlp.org/content/25/4/294.full.htmI\#ref-list-1
Articles cited in:
http://genesdev.cshlp.org/content/25/4/294.full.html\#related-urls

\section{License}
Email Alerting
Service
Receive free email alerts when new articles cite this article - sign up in the box at the top right corner of the article or click here.

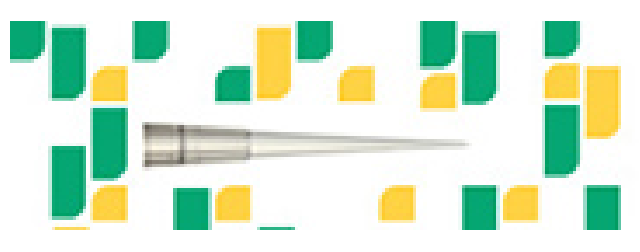

Focused on your science. 